\title{
On constructions of strong and uniformly minimal M-bases in Banach spaces
}

\author{
R. Vershynin* \\ 1991 Mathematics Subject Classification: 46B15.
}

\begin{abstract}
We find a natural class of transformations ("flattened perturbations") of a norming M-basis in a Banach space $X$, which give a strong norming M-basis in $X$. This simplifies and generalizes the positive answer to the "strong M-basis problem" solved by P. Terenzi. We also show that in general one cannot achieve uniformly minimality applying standard transformations to a given norming M-basis, despite of the existence in $X$ a uniformly minimal strong M-bases.
\end{abstract}

\section{Introduction}

Does every separable Banach space $X$ have a strong M-basis? This problem remained open for a long time (see [S], Problem 8.1) and was solved in positive by P. Terenzi ([T 90], [T 94]). He proved that every complete norming biorthogonal system has a block perturbation which is a strong complete norming biorthogonal system (a biorthogonal system $\left(z_{n}, z_{n}^{*}\right)_{n \geq 1}$ is a block perturbation of a biorthogonal system $\left(x_{n}, x_{n}^{*}\right)_{n \geq 1}$ if for every $m \geq 1$

$$
\left[z_{n}\right]_{n \in I(m)}=\left[x_{n}\right]_{n \in I(m)} \text { and } \quad\left[z_{n}^{*}\right]_{n \in I(m)}=\left[x_{n}^{*}\right]_{n \in I(m)}
$$

where $I(m), m=1,2, \ldots$, are some successive intervals of positive integers).

In Section 3 the way of constructing strong block perturbations is essentially simplified and slightly generalized. For a given complete norming biorthogonal system we find a certain class of block perturbations (so-called "flattened perturbations") which are strong norming complete biorthogonal systems. Therefore, we demonstrate a new construction of strong M-bases in every separable Banach space. The presentation of this part is self-contained.

The second part of the paper is concerned with questions of uniform minimality. A biorthogonal system $\left(z_{n}, z_{n}^{*}\right)_{n \geq 1}$ is called a pile perturbation of a biorthogonal system $\left(x_{n}, x_{n}^{*}\right)_{n \geq 1}$ if (1) holds for $m=1,2, \ldots$, where $I(m)$ are some intervals of integers with the left bounds $=1$ and the right bounds $\rightarrow \infty$ as $m \rightarrow \infty$. The notions of block and pile perturbations can be considered for minimal sequences as well: $\left(z_{n}\right)_{n \geq 1}$ is called a block (resp. pile) perturbation of $\left(x_{n}\right)_{n \geq 1}$ if we have

$$
\left[z_{n}\right]_{n \in I(m)}=\left[x_{n}\right]_{n \in I(m)}
$$

instead of (11) in the correspondent definitions. Clearly, each block perturbation is a pile perturbation. It was a long standing open problem in Banach space theory whether every separable Banach space $X$ has a uniformly minimal M-basis. R. Ovsepian and A. Pelczyński solved it in positive $([\overline{\mathrm{OP}}]$, see also $[\mathbb{P}])$. Later P. Terenzi constructed in every $X$ a strong uniformly minimal M-basis ([T 90], see [T 98] for further improvements). In view of the results of Section 3.2, these

\footnotetext{
${ }^{*}$ This research was finished when the author was visiting the Politecnic institute of Milan
} 
observations suggest the following question: can one achieve uniform minimality in constructions of strong block (or, at least, pile) perturbations?

The answer is in general negative. In Section 4 we construct a complete norming biorthogonal system $\left(x_{n}, x_{n}^{*}\right)_{n \geq 1}$ in $l_{2}$ without uniformly minimal pile perturbations. It follows that the norming M-basis $\left(x_{n}\right)_{n \geq 1}$ has no uniformly mininal block perturbations. The system $\left(x_{n}, x_{n}^{*}\right)_{n \geq 1}$ has even more pathological structure, to be established in Theorem 4.2.

I am grateful to V. Kadets for the guidance, and for P. Terenzi for his hospitality during my visit to Milan.

\section{Standard definitions}

Usual preliminaries can be found in [LT] and [S]. Nevertheless we recall some definitions. A system $\left(x_{n}, x_{n}^{*}\right)_{n \geq 1} \subset X \times X^{*}$ is called biorthogonal if $x_{n}^{*}\left(x_{m}\right)=\delta_{n, m}$ for every $n, m$ (Kronecker's delta). Suppose a complete sequence $\left(x_{n}\right)_{n \geq 1}$ is minimal, i.e. $x_{n} \notin\left[x_{m}\right]_{m \neq n}$ for any $n$. Then there exists a unique sequence of biorthogonal functionals $\left(x_{n}^{*}\right)_{n \geq 1} \subset X^{*}$, i.e. such that $\left(x_{n}, x_{n}^{*}\right)_{n \geq 1}$ is a biorthogonal system. A sequence $\left(x_{n}^{*}\right)_{n \geq 1} \subset X^{*}$ is called total if for every $x^{*} \in X^{*}$ one can find an $n$ so that $x_{n}^{*}(x) \neq 0$. Further, $\left(x_{n}^{*}\right)_{n \geq 1}$ is called norming if there exists a constant $c>0$ such that for every $x^{*} \in X^{*}$ one can find an $x \in\left[x_{n}\right]_{n \geq 1}$ with $\left|x_{n}^{*}(x)\right| \geq c\left\|x^{*}\right\|\|x\|$. Trivially every norming sequence is total. We will call a biorthogonal system itself $\left(x_{n}, x_{n}^{*}\right)_{n \geq 1}$ complete if the sequence $\left(x_{n}\right)_{n \geq 1}$ is complete, and total (resp. norming) if the sequence $\left(x_{n}^{*}\right)_{n \geq 1}$ is total (resp. norming).

A complete minimal system $\left(x_{n}\right)_{n \geq 1}$ is called an M-basis (resp. norming M-basis) if its sequence of biorthogonal functionals is total (resp. norming). A complete total biorthogonal system $\left(x_{n}, x_{n}^{*}\right)_{n \geq 1}$ (or simply an M-basis $\left(x_{n}\right)_{n \geq 1}$ ) is called strong if $x \in\left[x_{n}^{*}(x) x_{n}\right]_{n \geq 1}$ for every $x \in X$. There is an intrinsic characterization of strongness, due to A. Plans and A. Reyes [PR]: an M-basis $\left(x_{n}\right)_{n \geq 1}$ is strong iff $\left[x_{n}\right]_{n \in A} \cap\left[x_{n}\right]_{n \in B}=\left[x_{n}\right]_{n \in A \cap B}$ for every subsets of indices $A$ and $B$.

We say that a system $\left(x_{n}, x_{n}^{*}\right)_{n \geq 1} \subset X \times X^{*}$ (not necessarily biorthogonal) is $C$-bounded if $\left\|x_{n}\right\|\left\|x_{n}^{*}\right\| \leq C$ for every $n$. Clearly, a complete biorthogonal system $\left(x_{n}, x_{n}^{*}\right)_{n \geq 1}$ is $C$-bounded for some $C>0$ iff the sequence $\left(x_{n}\right)_{n \geq 1}$ is uniformly minimal, i.e. $\inf _{n} \operatorname{dist}\left(x_{n} /\left\|x_{n}\right\|,\left[x_{m}\right]_{m \neq n}\right)>0$ for every $n$. In this case we call the system $\left(x_{n}, x_{n}^{*}\right)_{n \geq 1}$ itself uniformly minimal.

\section{Strong block perturbations}

A partition of $\mathbf{N}$ into finite sets $(A(j))_{j \geq 1}$ is called a block partition if for some successive intervals of integers $(I(m))_{m \geq 1}$ the sets $\cup_{j \in I(m)} A(j)$ are successive intervals of integers, $m=1,2, \ldots$

We shall use the notion of block perturbations also for finite systems: $\left(z_{n}, z_{n}^{*}\right)_{n \leq m}$ is a block perturbation of $\left(x_{n}, x_{n}^{*}\right)_{n \leq m}$ if $\left[z_{n}\right]_{n \leq m}=\left[x_{n}\right]_{n \leq m}$ and $\left[z_{n}^{*}\right]_{n \leq m}=\left[x_{n}^{*}\right]_{n \leq m}$.

Let $\left(x_{n}, x_{n}^{*}\right)_{n \geq 1}$ be a biorthogonal system. Fix some partition of $\mathbf{N}$ into finite sets $(A(j))_{j \geq 1}$ and a sequence of numbers $n(j) \in A(j)$.

Definition 3.1 A biorthogonal system $\left(z_{n}, z_{n}^{*}\right)_{n \geq 1}$ is called a flattened perturbation of a biorthogonal system $\left(x_{n}, x_{n}^{*}\right)_{n \geq 1}$ with respect to $(n(j), A(j))$ if for every $j \geq 1$

(i) $\left(z_{n}, z_{n}^{*}\right)_{n \in A(j)}$ is a block perturbatoin of $\left(x_{n}, x_{n}^{*}\right)_{n \in A(j)}$;

(ii) $\left\|z_{n}^{*}-x_{n(j)}^{*}\right\| \leq \varepsilon_{j} /\left\|x_{n(j)}\right\|$ for $n \in A(j)$,

where $\varepsilon_{j}$ are some positive scalars with $\sum \varepsilon_{j}<\infty$.

Trivially, if $(A(j))$ is a block partition, then every flattened perturbation with respect to $(n(j), A(j))$ is a block perturbation. Note that flattened perturbations are easy to construct: one can apply an invertible linear operator acting in $\left[x_{n}^{*}\right]_{n \in A(j)}$ which sends each $x_{n}^{*}$ to some vector close to $x_{n(j)}^{*}$. 
Now we state the main result in this section.

Theorem 3.2 Let $\left(x_{n}, x_{n}^{*}\right)_{n \geq 1}$ be a complete norming biorthogonal system. Then there is a block partition $(A(j))$ and numbers $n(j) \in A(j)$ so that each flattened perturbation of $\left(x_{n}, x_{n}^{*}\right)_{n \geq 1}$ with respect to $(n(j), A(j))$ is a strong complete biorthogonal system.

We wil use the following two known results, due to P. Terenzi. Since their proofs are scattered among different papers, and for the sake of completeness, we prove these results below.

Lemma 3.3 Let $\left(x_{n}, x_{n}^{*}\right)_{n \geq 1}$ be a complete biorthogonal system in a Banach space $X$. Then there is a sequence of positive integers $r(1)<r(2)<\ldots$ (which we call representing indices) so that for every $x \in X$

$$
x=\lim _{m}\left(\sum_{n=1}^{r(m)} x_{n}^{*}(x) x_{n}+v_{m}\right)
$$

for some vectors $v_{m} \in\left[x_{n}\right]_{n=r(m)+1}^{r(m+1)}$ depending on $x$.

Lemma 3.4 If $\left(x_{n}, x_{n}^{*}\right)_{n \geq 1}$ is a complete norming biorthogonal system in $X$, then representing indices can be chosen with the following property. Suppose for some $x \in X$ there is a sequence of positive integers $m_{1}<m_{2}<\ldots$ so that

$$
\text { the series } \sum_{k=1}^{\infty} \sum_{n=r\left(m_{k}\right)+1}^{r\left(m_{k}+1\right)} x_{n}^{*}(x) x_{n} \quad \text { converges. }
$$

Then setting $r\left(m_{0}\right)=0$ we have

$$
x=\sum_{k=0}^{\infty} \sum_{n=r\left(m_{k}\right)+1}^{r\left(m_{k+1}\right)} x_{n}^{*}(x) x_{n} .
$$

In the sequel, the relation $x \underset{\varepsilon}{\approx} y$ between two vectors $x$ and $y$ means that $\|x-y\| \leq \varepsilon$. We also assume for convenience that $\sum_{n \in \emptyset} y_{n}=0$ for every vectors $y_{n}$.

Proof of Lemma 3.3. We proceed by induction. Set $r(1)=1$ and assume that $(r(n))_{n \leq m}$ is constructed for some $m \geq 1$. Then, by a simple compactness argument (hint: a finite net) there exists a number $p(m+1)>r(m)$ large enough so that for every $z \in\left[x_{n}\right]_{n \leq r(m)}$ with $\|z\|=1$

$$
\operatorname{dist}\left(z,\left[x_{n}\right]_{n=r(m)+1}^{p(m+1)}\right) \stackrel{\delta}{\approx} \operatorname{dist}\left(z,\left[x_{n}\right]_{n=r(m)+1}^{\infty}\right),
$$

where $\delta$ can be taken sufficiently small: $\delta=\left(m \sum_{n \leq r(m)}\left\|x_{n}^{*}\right\|\left\|x_{n}\right\|\right)^{-1}$. Now set $r(m+1):=p(m+1)$.

Let us check that $(r(n))$ is, indeed, a sequence of representing indices. Let $x \in B(X)$ and $\varepsilon \in(0,1)$. If a number $m$ is sufficiently large, then there is an $\widehat{x} \in\left[x_{n}\right]_{n \leq r(m)}$ with $\widehat{x} \underset{\approx}{\approx}$. Then (44) holds for $z:=\widehat{x}-\sum_{n \leq r(m)} x_{n}^{*}(x) x_{n}$ with $\delta=\varepsilon$. But $z \stackrel{\varepsilon}{\approx} x-\sum_{n \leq r(m)} x_{n}^{*}(x) x_{n}=: x^{\prime}$; hence $\operatorname{dist}\left(x^{\prime},\left[x_{n}\right]_{n=r(m)+1}^{p(m+1)}\right) \stackrel{3 \varepsilon}{\approx} \operatorname{dist}\left(x^{\prime},\left[x_{n}\right]_{n=r(m)+1}^{\infty}\right)=0$. Then $x^{\prime}$ is within a distance $3 \varepsilon$ from $\left[x_{n}\right]_{n=r(m)+1}^{p(m+1)}=\left[x_{n}\right]_{n=r(m)+1}^{r(m+1)}$. This completes the proof. 
Proof of Lemma 3.4. Assume $(r(n))_{n \leq m}$ is constructed for some $m \geq 0$. As in the proof of Lemma 3.3, we find a number $p(m+1)>r(m)$ so that (四) holds for every $z \in\left[x_{n}\right]_{n \leq r(m)}$ with $\|z\|=1$. Recall that the biorthogonal system $\left(x_{n}, x_{n}^{*}\right)_{n \geq 1}$ is $(2 c)$-norming for some $c>0$. That is, given a $v \in X$, we have $x^{*}(v) \geq 2 c\|v\|$ for some $x^{*} \in\left[x_{n}^{*}\right]_{n \geq 1}$ with $\left\|x_{n}^{*}\right\|=1$. The simple compactness argument provides a number $r(m+1)>p(m+1)$ such that we have the following property:

(P) If $v \in\left[x_{n}\right]_{n \leq p(m+1)}$, then $x^{*}(v) \geq c\|v\|$ for some $x^{*} \in\left[x_{n}^{*}\right]_{n \leq r(m+1)}$ with $\left\|x^{*}\right\|=1$.

Then $(r(n))$ constructed in this way is, indeed, a sequence of representing indices. Let us verify that the conclusion of Lemma 3.4 holds. Substracting the convergent series, we can assume that for every $k \geq 1$

$$
x_{n}^{*}(x)=0 \quad \text { whenever } \quad r\left(m_{k}\right)+1 \leq n \leq r\left(m_{k}+1\right) .
$$

We can also assume that $x \in B(X)$. Let $\varepsilon>0$; by the proof of Lemma 3.3 we have for any sufficiently large integer $k$

$$
x \stackrel{\varepsilon}{\approx} \sum_{n \leq r\left(m_{k}\right)} x_{n}^{*}(x) x_{n}+v_{m_{k}} \quad \text { with } \quad v_{m_{k}} \in\left[x_{n}\right]_{n=r\left(m_{k}\right)+1}^{p\left(m_{k}+1\right)} .
$$

Therefore, to finish the proof it is enough to show that $\lim _{k} v_{m_{k}}=0$.

Using (可), we get $v_{m_{k}} \stackrel{\varepsilon}{\approx} x-\sum_{n \leq r\left(m_{k}+1\right)} x_{n}^{*}(x) x_{n}$. Therefore $x^{*}\left(v_{m_{k}}\right) \stackrel{\varepsilon}{\approx} 0$ for any $x^{*} \in$ $\left[x_{n}^{*}\right]_{n \leq r\left(m_{k}+1\right)}$ with $\left\|x^{*}\right\|=1$. On the other hand, by the property $(\mathrm{P})$, we have $x^{*}\left(v_{m_{k}}\right) \geq c\left\|v_{m_{k}}\right\|$ for some $x^{*} \in\left[x_{n}^{*}\right]_{n \leq r\left(m_{k}+1\right)}$ with $\left\|x^{*}\right\|=1$. These estimates yield $c\left\|v_{m_{k}}\right\| \leq \varepsilon$. This completes the proof.

Proof of Theorem 3.2. Let $(r(m))$ be representing indices of $\left(x_{n}, x_{n}^{*}\right)_{n \geq 1}$. We construct the block partition $(A(j))$ and numbers $n(j) \in A(j)$ by induction. At each successive step, we find a successive interval of integers ending at some representing index $r(m)$, and this interval will be the new block of sets $A(j)$. Suppose $(n(j), A(j))_{j \leq j_{0}}$ is constructed and let $r(m)$ be the last element of the interval $\cup_{j \leq j_{0}} A(j)$. We call such $r(m)$ a block bound.

For $r(m)+1 \leq j \leq r(m+1)$, let $d_{j}=m+j-r(m)$. Let $E(j)$ be the set consisting of $\{j\}$ plus the successive interval between representing indices:

$$
E(j)=\{j\} \cup\left\{r\left(d_{j}\right)+1, \ldots, r\left(d_{j}+1\right)\right\} .
$$

We see that the sets $E(j)$ are disjoint and their union is an interval beginning at $r(m)+1$. These $E(j)$ will form the new block of sets $A(j)$. More precisely, we define

$$
A\left(j_{0}+j-r(m)\right)=E(j) \quad \text { and } \quad n\left(j_{0}+j-r(m)\right)=j
$$

for $r(m)+1 \leq j \leq r(m+1)$. This completes the construction.

Observe that the set $\{n(1), n(2), \ldots\}$ is exactly the union of the intervals $\{r(m)+1, \ldots, r(m+1)\}$, where $r(m)$ are the block bounds. Let $j(n)$ be the (one-to-one) function from this set to $\mathbf{N}$ which $\operatorname{maps} n(j)$ to $j$.

Now we verify the conclusion of Theorem 3.2. Let $\left(z_{n}, z_{n}^{*}\right)_{n \geq 1}$ be any flattened perturbation of $\left(x_{n}, x_{n}^{*}\right)_{n \geq 1}$ with respect to $(n(j), A(j))$, and let $\sum \varepsilon_{n}$ be a convergent series of positive numbers. Pick any $x \in S(X)$. We are to show that $x \in\left[z_{n}^{*}(x) z_{n}\right]_{n \geq 1}$. There are two possibilities:

(A) There exists a sequence of block bounds $r\left(m_{1}\right)<r\left(m_{2}\right)<\ldots$ such that for every $k \geq 1$

$$
\left\|x_{n}^{*}(x) x_{n}\right\| \leq \varepsilon_{j(n)}
$$

for $r\left(m_{k}\right)+1 \leq n \leq r\left(m_{k}+1\right)$; 
(B) For every sufficiently large block bound $r(m)$ there is a $n_{0}=n_{0}(m)$ with $r(m)+1 \leq n_{0} \leq$ $r(m+1)$ such that

$$
\left\|x_{n_{0}}^{*}(x) x_{n_{0}}\right\|>\varepsilon_{j\left(n_{0}\right)} \quad \text { and } \quad\left\|x_{n}^{*}(x) x_{n}\right\| \leq \varepsilon_{j(n)}
$$

for $n_{0} \leq n \leq r(m+1)$.

If $(\mathrm{A})$ is the case, then the convergence of the series $\sum_{k=1}^{\infty} \sum_{n=r\left(m_{k}\right)+1}^{r\left(m_{k}+1\right)} \varepsilon_{j(n)} \leq \sum_{j=1}^{\infty} \varepsilon_{j}$ makes possible to apply Lemma 3.4. We derive from (3) that $x \in\left[z_{n}^{*}(x) z_{n}\right]_{n \geq 1}$, since $\left(z_{n}, z_{n}^{*}\right)_{n=r\left(m_{k}\right)+1}^{r\left(m_{k+1}\right)}$ is a block perturbation of $\left(x_{n}, x_{n}^{*}\right)_{n=r\left(m_{k}\right)+1}^{r\left(m_{k+1}\right)}$. This completes the proof in this case.

If (B) is the case, then another argument works. Consider the set $\Omega=\left\{n \in \mathbf{N}: z_{n}^{*}(x)=0\right\}$. It is enough to show that $x \in\left[z_{n}\right]_{n \in \mathbf{N} \backslash \Omega}$. Fix an $\varepsilon>0$ and a sufficiently large block bound $r(m)$; let $n_{0}$ be an index guaranteed by $(\mathrm{B})$.

CLAIM: $\left.E\left(n_{0}\right)\right) \subset \mathbf{N} \backslash \Omega$.

Indeed, it follows from (6) that $E\left(n_{0}\right)=A\left(j\left(n_{0}\right)\right)$. If the Claim were not true, then $z_{n}^{*}(x)=0$ for some $n \in A\left(j\left(n_{0}\right)\right)$. Then by the definition of a flattened perturbation we would have $\left|x_{n_{0}}^{*}(x)\right| \leq$ $\varepsilon_{j\left(n_{0}\right)} /\left\|x_{n_{0}}\right\|$, which contradicts to (7).

By Lemma 3.3, there is a vector $v \in\left[x_{n}\right]_{n \in E\left(n_{0}\right)}$ such that setting $\Gamma=\{1, \ldots, r(m)\} \cup\left(\cup_{j=r(m)+1}^{n_{0}-1}\right.$ $E(j))$ we have

$$
\begin{aligned}
x & \stackrel{\varepsilon}{\approx} \sum_{n=1}^{r\left(d_{n_{0}}\right)} x_{n}^{*}(x) x_{n}+v \\
& =\sum_{n \in \Gamma} x_{n}^{*}(x) x_{n}+\left(x_{n_{0}}^{*}(x) x_{n_{0}}+v\right)+\sum_{n=n_{0}+1}^{r(m+1)} x_{n}^{*}(x) x_{n} .
\end{aligned}
$$

The first summand belongs to $\left[z_{n}\right]_{n \in \mathbf{N} \backslash \Omega}$. Indeed, $\left(x_{n}, x_{n}^{*}\right)_{n \in \Gamma}$ is a block perturbation of $\left(z_{n}, z_{n}^{*}\right)_{n \in \Gamma}$; thus $\sum_{n \in \Gamma} x_{n}^{*}(x) x_{n}=\sum_{n \in \Gamma} z_{n}^{*}(x) z_{n} \in\left[z_{n}\right]_{n \in \mathbf{N} \backslash \Omega}$. The second summand belongs to $\left[x_{n}\right]_{n \in E\left(n_{0}\right)}=$ $\left[z_{n}\right]_{n \in E\left(n_{0}\right)} \subset\left[z_{n}\right]_{n \in \mathbf{N} \backslash \Omega}$ by the Claim. The third summand has the norm less than $\varepsilon$ if $m$ and, therefore, $n_{0}=n_{0}(m)$, were chosen sufficiently large: this follows from (7).

Thus we have shown that $\operatorname{dist}\left(x,\left[x_{n}\right]_{n \in \mathbf{N} \backslash \Omega}\right)<2 \varepsilon$. This completes the proof.

\section{Uniformly minimal pile perturbations}

We shall find a biorthogonal system $\left(x_{n}, x_{n}^{*}\right)_{n \geq 1}$ in $l_{2}$ which has no uniformly minimal pile perturbations. This will follow from a more general result.

Definition 4.1 We say that a biorthogonal system $\left(z_{n}, z_{n}^{*}\right)_{n \geq 1}$ is spanned by a biorthogonal system $\left(x_{n}, x_{n}^{*}\right)_{n \geq 1}$ if

$$
\left(z_{n}\right)_{n \geq 1} \subset \operatorname{span}\left(x_{n}\right)_{n \geq 1} \quad \text { and } \quad\left(z_{n}^{*}\right)_{n \geq 1} \subset \operatorname{span}\left(x_{n}^{*}\right)_{n \geq 1} .
$$

If (8) holds, then to every positive integer $m \geq 1$ we can assign the minimal number $q(m)$ such that

$$
\left[z_{n}\right]_{n \leq m} \subset\left[x_{n}\right]_{n \leq q(m)} \text { and }\left[z_{n}^{*}\right]_{n \leq m} \subset\left[x_{n}^{*}\right]_{n \leq q(m)} .
$$

We call $(q(m))_{m \geq 1}$ the spanning indices. Obviously, $q(m) \geq m$ for every $m \geq 1$. Clearly, $\left(z_{n}, z_{n}^{*}\right)_{n \geq 1}$ is a pile perturbation of $\left(x_{n}, x_{n}^{*}\right)_{n \geq 1}$ iff the equality $q(m)=m$ holds for infintely many positive integers $m$.

The main result in this section states that there are complete norming biorthogonal systems in $l_{2}$ such that uniformly minimal systems spanned by them must have very large spanning indices. 
Theorem 4.2 Given a sequence of positive numbers $\left(\lambda_{m}\right)_{m \geq 1}$, there is a complete biorthogonal system $\left(x_{n}, x_{n}^{*}\right)_{n \geq 1}$ in $l_{2}$ with the following property. If $(q(m))$ are the spanning indices of a uniformly minimal system spanned by $\left(x_{n}, x_{n}^{*}\right)_{n \geq 1}$, then

$$
\lim _{m} q(m) / \lambda_{m}=\infty .
$$

Corollary 4.3 There exists a complete norming biorthogonal system $\left(x_{n}, x_{n}^{*}\right)_{n \geq 1}$ in $l_{2}$ without uniformly minimal pile and block perturbations. Moreover, the norming M-basis $\left(x_{n}\right)_{n \geq 1}$ has no uniformly minimal block perturbations.

Proof. The first statement follows from Theorem 4.2 if we set $\lambda_{m}=m, m=1,2, \ldots$

Let $\left(z_{n}\right)_{n \geq 1}$ be arbitrary block perturbation of $\left(x_{n}\right)_{n \geq 1}$. Let $I(m)$ be successive intervals of integers so that (2) holds for every $m$. Then

$$
\left[x_{n}^{*}\right]_{n \in I(m)}=\left(\left[x_{n}\right]_{n \in I\left(m^{\prime}\right), m^{\prime} \neq m}\right)^{\perp}=\left(\left[z_{n}\right]_{n \in I\left(m^{\prime}\right), m^{\prime} \neq m}\right)^{\perp}=\left[z_{n}^{*}\right]_{n \in I(m)} .
$$

Hence $\left(x_{n}, x_{n}^{*}\right)_{n \geq 1}$ is a block perturbation of $\left(z_{n}, z_{n}^{*}\right)_{n \geq 1}$. Then it follows from the first part that $\left(x_{n}\right)_{n \geq 1}$ is not uniformly minimal.

Remarks. 1. Of course, $\left(x_{n}\right)_{n \geq 1}$ has a uniformly minimal pile perturbation (apply the standard biorthogonalization procedure in $l_{2}$ ).

2. It will follow from the proof that these results hold not only in $l_{2}$, but also in every reflexive Banach space with unconditional basis.

We proceed now to the proof of Theorem 4.2.

Let $\left(e_{n}\right)_{n \geq 1}$ denote the canonical basis in $l_{2}$. Let $\pi$ be some permutation on $\mathbf{N}$. We shall specify $\pi$ later, it will depend only on the sequence $\left(\lambda_{n}\right)_{n \geq 1}$. Let $\left(\varepsilon_{n}\right)_{n \geq 1}$ be a sequence of positive numbers such that

$$
\sum_{i>n} \varepsilon_{i}^{2} \leq 1 / 8
$$

The following proposition can be derived easily from the standard construction of an M-basis in a separable Banach space (see [LT], Proposition 1.f.3).

Proposition 4.4 There is a biorthogonal system $\left(x_{n}, x_{n}^{*}\right)_{n \geq 1}$ in $l_{2}$ and a sequence $\left(\widehat{e}_{n}\right)_{n \geq 1}$ in $l_{2}$ such that:

(i) $\left[x_{n}\right]_{n \leq m}=\left[\widehat{e}_{n}\right]_{n \leq m}$ and $\left[x_{n}^{*}\right]_{n \leq m}=\left[e_{\pi(n)}\right]_{n \leq m}$ for every $m \geq 1$;

(ii) $\widehat{e}_{n} \stackrel{\varepsilon_{n}}{\approx} e_{n}$ for every $n \geq 1$.

Apply Proposition 4.4 and define a linear operator $T$ in $l_{2}$ by

$$
T \widehat{e}_{n}=e_{n}, \quad n \geq 1 \text {. }
$$

It is not hard to check that (9) yields that $T$ is well defined and is an isomorphism:

$$
\|T\| \leq 2, \quad\left\|T^{-1}\right\| \leq 2 .
$$

In particular, $\left(\widehat{e}_{n}\right)_{n \geq 1}$ is a basis in $l_{2}$. Then $\left(x_{n}\right)_{n \geq 1}$ and $\left(x_{n}^{*}\right)_{n \geq 1}$ are complete sequences. Hence $\left(x_{n}, x_{n}^{*}\right)_{n \geq 1}$ is a complete norming biorthogonal system.

It will not be enough to know that $T$ is just an isomorphism. The following lemma shows that $T$ is asymptotically close to the identity. 
Lemma 4.5 Let $\left(z_{n}\right)_{n \geq 1}$ be a normalized $M$-basis in $l_{2}$. Then

$$
\lim _{n}\left(T z_{n}-z_{n}\right)=0 .
$$

Proof. It suffices to show that $\lim _{n}\left\|z_{n}-T^{-1} z_{n}\right\|=0$. Let $\varepsilon>0$. Let $n_{0}=n_{0}(\varepsilon)$ be a positive integer which we specify below. For every $n \geq 1$, write the expansions $z_{n}=\sum_{i} a_{n, i} e_{i}$, where $\left(a_{n, i}\right)$ are some scalars. By the triangle inequality, Hölder's inequality, and our choise of vectors $\widehat{e}_{i}$, we have for any $k \geq 1$

$$
\begin{aligned}
\left\|z_{n}-T^{-1} z_{n}\right\| & =\left\|\sum_{i \geq 1} a_{n, i}\left(e_{i}-\widehat{e}_{i}\right)\right\| \\
& \leq \sum_{i \leq k}\left|a_{n, i}\right|+\left(\sum_{i>k}\left|a_{n, i}\right|^{2}\right)^{1 / 2}\left(\sum_{i>k}\left\|e_{i}-\widehat{e}_{i}\right\|^{2}\right)^{1 / 2} \\
& \leq \sum_{i \leq k}\left|a_{n, i}\right|+\left(\sum_{i>k} \varepsilon_{i}^{2}\right)^{1 / 2}
\end{aligned}
$$

CLAIM: $\lim _{n} a_{n, i}=0$ for every $i \geq 1$.

Indeed, since $\left(z_{n}\right)_{n \geq 1}$ is a normalized M-basis in a reflexive space, the sequence $\left(z_{n}\right)_{n \geq 1}$ tends weakly to zero. Then for every $i \geq 1$

$$
0=\lim _{n}\left\langle e_{i}, z_{n}\right\rangle=\lim _{n} a_{n, i}
$$

This proves the Claim.

Now we describe how to pick $n_{0}$. First choose $k=k(\varepsilon)$ so that the second summand in (11) is less then $\varepsilon / 2$. By Claim, we can pick $n_{0}=n_{0}(k, \varepsilon)$ so that the first summand in (11) is less then $\varepsilon / 2$ whenever $n>n_{0}$. Thus $\left\|z_{n}-T^{-1} z_{n}\right\|<\varepsilon / 2+\varepsilon / 2=\varepsilon$ for every $n>n_{0}$.

We proceed to verification of the conclusion of Theorem 4.2. Let $\left(z_{n}, z_{n}^{*}\right)_{n \geq 1}$ be a system spanned by $\left(x_{n}, x_{n}^{*}\right)_{n \geq 1}$, and such that

$$
\left\|z_{n}\right\|=1, \quad\left\|z_{n}^{*}\right\| \leq M \quad \text { for } \quad n \geq 1
$$

Assume that the conclusion of Theorem 4.2 is false. Then there are a positive (integer) constant $c$ and increasing sequences of positive integers $(p(m))_{m \geq 1}$ and $(q(m))_{m \geq 1}$ such that for every $m \geq 1$ we have:

$$
r(m):=q(p(m)) \leq c \lambda_{p(m)}
$$

and

$$
\begin{gathered}
{\left[z_{n}\right]_{n \leq p(m)} \subset\left[x_{n}\right]_{n \leq r(m)}=\left[\widehat{e}_{n}\right]_{n \leq r(m)},} \\
{\left[z_{n}^{*}\right]_{n \leq p(m)} \subset\left[x_{n}^{*}\right]_{n \leq r(m)}=\left[e_{\pi(n)}\right]_{n \leq r(m)} .}
\end{gathered}
$$

For a positive integer $k$, set

$$
\Omega(k)=\{1,2, \ldots, k\} \cap\{\pi(1), \pi(2), \ldots, \pi(k)\} .
$$

Note that if $k$ is large enough, then $\Omega(k)$ is not empty.

For any integer $m$ large enough, let $P_{m}$ denote the orthogonal projection in $l_{2}$ onto $\left[e_{n}\right]_{n \in \Omega(r(m))}$. Define a system $\left(y_{m, n}, y_{m, n}^{*}\right)_{n \leq p(m)}$ by

$$
y_{m, n}=P_{m} T z_{n}, \quad y_{m, n}^{*}=P_{m} z_{n}^{*} .
$$

By (10) and (12), this system is $(2 M)$-bounded. One can not assert that it is biorthogonal, but this is not far from truth. 
Definition 4.6 Let $X$ be a Banach space and $\varepsilon>0$. A system $\left(y_{n}, y_{n}^{*}\right) \subset X \times X^{*}$ (finite or infinite) is called $\varepsilon$-roughly biorthogonal in $X$ if, for every indices $k$ and $n, y_{k}^{*}\left(y_{n}\right) \stackrel{\varepsilon}{\approx} \delta_{k, n}$.

Lemma 4.7 There are positive integers $n_{0}$ and $m_{0}$ such that, for every $m>m_{0}$, the system $\left(y_{m, n}, y_{m, n}^{*}\right)_{n=n_{0}+1}^{p(m)}$ is a $(1 / 4)$-roughly biorthogonal system in $l_{2}$.

Proof. Lemma 4.5 provides a number $n_{0}$ such that

$$
\left\|T z_{n}-z_{n}\right\|<\frac{1}{4 M} \quad \text { for } \quad n>n_{0}
$$

Pick $m_{0}$ so that $\Omega\left(r\left(m_{0}\right)\right)$ is not empty and $p\left(m_{0}\right)>n_{0}$. Fix a positive integer $m>m_{0}$ and take any indices $n$ and $k$ with $n_{0}+1 \leq n, k \leq p(m)$. By (14) and (15),

$$
\operatorname{supp}\left(z_{k}^{*}\right) \cap \operatorname{supp}\left(T z_{n}\right) \subset \Omega(r(m)) .
$$

Then

$$
y_{m, k}^{*}\left(y_{m, n}\right)=\left\langle P_{m} z_{k}^{*}, P_{m} T z_{n}\right\rangle=z_{k}^{*}\left(T z_{n}\right) .
$$

Together with (16) and (12), this gives

$$
\left|y_{m, k}^{*}\left(y_{m, n}\right)-\delta_{k, n}\right|=\left|z_{k}^{*}\left(T z_{n}-z_{n}\right)\right| \leq\left\|z_{k}^{*}\right\| \cdot \frac{1}{4 M} \leq 1 / 4 .
$$

The proof is complete.

Observe that $\operatorname{supp}\left(y_{m, n}\right) \subset \Omega(r(m))$ and $\operatorname{supp}\left(y_{m, n}^{*}\right) \subset \Omega(r(m))$ for every $m>m_{0}$ and $n \leq$ $p(m)$. Therefore, for every $m>m_{0}$ we may consider the vectors $y_{m, n}$ and $y_{m, n}^{*}, n \leq p(m)$, as elements of the space $l_{2}^{|\Omega(r(m))|}$.

Summarize what we have shown. There are positive integers $n_{0}$ and $m_{0}$ such that, for every integer $m>m_{0}$, there is a system of cardinality $\left(p(m)-n_{0}\right)$ in $l_{2}^{|\Omega(r(m))|}$ which is:

(i) (1/4)-roughly biorthogonal,

(ii) $(2 M)$-bounded.

Now we establish that in this case $|\Omega(r(m))|$ can not be too large.

Lemma 4.8 Let $\varepsilon \in(0,1 / 2)$. Let $X$ be a $k$-dimensional Banach space. Suppose some system $\left(y_{n}, y_{n}^{*}\right)_{n \leq p}$ in $X$ is $\varepsilon$-roughly biorthogonal and $M$-bounded. Then

$$
k \geq c_{1} \log p
$$

for some constant $c_{1}=c_{1}(\varepsilon, M)>0$.

Proof. We may assume $\left\|y_{n}\right\|=1$ and $\left\|y_{n}^{*}\right\| \leq M$ for all $n$. Then, for any non-equal indices $k$ and $n$, one has

$$
\left\|y_{k}-y_{n}\right\| \geq\left(y_{k}^{*}\left(y_{k}\right)-y_{k}^{*}\left(y_{n}\right)\right) /\left\|y_{k}^{*}\right\| \geq(1-2 \varepsilon) / M=: \delta .
$$

This shows that the open balls $y_{n}+(\delta / 2) B(X), n \leq p$, are pairwise disjoint and are contained in the ball $(1+\delta / 2) B(X)$. By comparing the volumes we get $p(\delta / 2)^{k} \leq(1+\delta / 2)^{k}$. Hence $k \geq(\log (1+2 / \delta))^{-1} \log p$. This completes the proof. 
Applying Lemma 4.8 in our situation, we obtain that there is a constant $c_{1}=c_{1}(M)$ such that, for every $m>m_{0}$,

$$
|\Omega(r(m))| \geq c_{1} \log \left(p(m)-n_{0}\right)
$$

Combining with (13), we have for $m>m_{0}$ :

$$
\left|\Omega\left(c \lambda_{p(m)}\right)\right| \geq c_{1} \log \left(p(m)-n_{0}\right)
$$

(clearly, we can assume that $\left(\lambda_{m}\right)_{m \geq 1}$ is an increasing sequence of positive integers). Since the sequence $(p(m))_{m \geq 1}$ is increasing, we get

$$
\limsup _{n} \frac{\left|\Omega\left(c \lambda_{n}\right)\right|}{\log n}>0
$$

Now we show that there is a permutation $\pi$ on $\mathbf{N}$ such that (17) fails for any constant $c$.

Lemma 4.9 Let $f: \mathbf{N} \rightarrow \mathbf{R}_{+}$be a non-decreasing function with $\lim _{n} f(n)=\infty$. Then there is a permutation $\pi$ on $\mathbf{N}$ with

$$
\lim _{n} \frac{|\Omega(c n)|}{f(n)}=0
$$

for every (positive integer) constant c.

Proof. One can easily construct a non-decreasing "onto" function $\varphi: \mathbf{N} \rightarrow \mathbf{N}$ such that

(i) $\lim _{n} \varphi(n)=\infty$;

(ii) $\lim _{n} \varphi(n) / f(n)=0$;

(iii) $\varphi(n) \leq n$ and $\varphi(2 n) \leq 2 \varphi(n)$ for every $n \geq 1$.

Then we define a function $\Phi: \mathbf{N} \rightarrow \mathbf{N}$ :

$$
\Phi(n)=|\{m: \varphi(m) \leq n\}| .
$$

Note that, for every $m \geq 1$,

$$
|\{n: \Phi(n) \leq m\}|=\varphi(m) .
$$

Let $\Gamma$ be a subset of $\mathbf{N}$ such that for every $m \geq 1$

$$
|\Gamma \cap\{1, \ldots, m\}| \leq \varphi(m) .
$$

Finally, define a permutation $\pi$ as follows:

$$
\pi(n)= \begin{cases}\Phi(n), & n \notin \Gamma \\ \operatorname{free}(n), & n \in \Gamma,\end{cases}
$$

where free $(n)$ denotes the minimal positive integer $k \notin\{\pi(1), \pi(2), \ldots, \pi(n-1)\}$. The permutation $\pi$ is well defined: indeed, the functions $\Phi(n)$ and free $(n)$ are strictly increasing and $\Phi(n) \geq n$, free $(n) \leq n$ for every $n$. We see that

$$
\Omega(m) \subset\{n: \Phi(n) \leq m\} \cup\{\text { free }(n): n \in \Gamma \cap\{1, \ldots, m\}\} .
$$

By (18) and (19),

$$
|\Omega(m)| \leq 2 \varphi(m) .
$$

Let a positive integer $k$ be so that $2^{k} \geq c$. By (20) and (iii), we have for every $n \geq 1$ :

$$
|\Omega(c n)| \leq\left|\Omega\left(2^{k} n\right)\right| \leq 2 \varphi\left(2^{k} n\right) \leq 2 \cdot 2^{k} \varphi(n) .
$$

Thus

$$
\lim _{n} \frac{|\Omega(c n)|}{f(n)} \leq 2^{k+1} \lim _{n} \frac{\varphi(n)}{f(n)}=0
$$

The proof is complete. 


\section{References}

[LT] J. Lindenstrauss, L. Tzafriri, Classical Banach spaces, Springer, 1977.

[MS] V. Milman, G. Schechtman, Asymptotic theory of finite dimensional normed spaces, Lecture Notes in Math., vol. 1200, Springer Verlag, 1986.

[OP] R. I. Ovsepian and A. Pelczyński, On the existence of a fundamental total and bounded sequence in every separable Banach space, and related constructions of uniformly bounded orthonormal systems in $L^{2}$, Studia Math. 54 (1975), 149-159

[P] A. Pelczyński, All separable Banach spaces admit for every $\varepsilon>0$ fundamental total and bounded by $1+\varepsilon$ biorthogonal sequences, Studia Math. 55 (1976), 295-304

[PR] A. Plans and A. Reyes, On the geometry of sequences in Banach spaces, Arch. Math. (Basel) 40 (1983), 452-458

[S] Singer, Bases in Banach spaces II, Springer Verlag, 1981.

[T 87] P. Terenzi, On the theory of fundamental norming bounded biorthogonal systems in Banach spaces, Trans. Amer. Math. Soc. 299 (1987), 497-511.

[T 90] P. Terenzi, Every norming M-basis of a separable Banach space has a block-perturbation which is norming strong M-basis, Extracta Mathematicae, España (1990), 161-169.

[T 94] P. Terenzi, Every separable Banach space has a bounded strong norming biorthogonal sequence which is also a Steinitz basis, Studia Math 111 (1994), 207-222.

[T 98] P. Terenzi, A positive answer to the basis problem, Israel J. Math. 104 (1998), 51-124.

e-mail addresses: paoter@mate.polimi.it, boris.v.novikov@univer.kharkov.ua 